\title{
Gravitational Waves in the Spectral Action of Noncommutative Geometry
}

\author{
William Nelson*, Joseph Ochoa ${ }^{\dagger}$ \\ Institute of Gravitation and the Cosmos, Penn State University, State College, PA 16801, U.S.A. \\ Mairi Sakellariadou $\ddagger$
Department of Physics, King's College, University of London, Strand WC2R 2LS, London, U.K.
}

The spectral triple approach to noncommutative geometry allows one to develop the entire standard model (and supersymmetric extensions) of particle physics from a purely geometry stand point and thus treats both gravity and particle physics on the same footing. The bosonic sector of the theory contains a modification to Einstein-Hilbert gravity, involving a nonconformal coupling of curvature to the Higgs field and conformal Weyl term (in addition to a nondynamical topological term). In this paper we derive the weak field limit of this gravitational theory and show that the production and dynamics of gravitational waves are significantly altered. In particular, we show that the graviton contains a massive mode that alters the energy lost to gravitational radiation, in systems with evolving quadrupole moment. We explicitly calculate the general solution and apply it to systems with periodically varying quadrupole moments, focusing in particular on the the well know energy loss formula for circular binaries.

PACS numbers: 11.10.Nx, 04.50.+h, 12.10.-g, 11.15.-q, 12.10.Dm

\section{INTRODUCTION}

Noncommutative Geometry (NCG) is a gravitational theory which, even in its simplest form, can explain the Standard Model of particle physics, and account for all current experimental data, in a rather simple and certainly elegant way. The simple - in the sense that it generalizes the continuum Riemaniann manifold by considering its product by a discrete two points space NCG proposal should be certainly replaced by a less trivial noncommutative space as one reaches Planckian energy scales. Nevertheless, this is the proposal we have at hand, and given its success in accounting for the Standard Model of particle physics, it offers a valid theoretical framework to address early universe issues. Alternatively, one can use experimental high energy physics data and astrophysical observations/measurements in order to test this NCG proposal and constrain its parameters. This is the approach used in this study.

One should indeed view this NCG proposal as an effective theory, which can however offer a valuable information about any NCG approach. In what follows we derive explicitly the weak field limit of this gravitational theory and then show that the production and dynamics of gravitational waves are both considerably modified from those obtained within the familiar General Relativity approach.

More precisely, in Section [I] we give a short introduction to the noncommutative geometry spectral action, the framework within which we will then focus. In Section III we first state in detail the conventions and sig-

\footnotetext{
*nelson@gravity.psu.edu

†jro166@psu.edu

$\ddagger$ mairi.sakellariadou@kcl.ac.uk
}

nature we use and we analyze the issue of gauge conditions. We then analyze linear perturbations around a Minkowski background metric and we solve the noncommutative geometry gravitational wave equation in terms of the retarded Green's function. We find that gravitational waves are only sourced from systems with a nontrivial quadrupole moment, as within General Relativity, while the NCG theory contains massive as well as massless gravitons. In Section[IV we concentrate first on some simple and then on some physical examples. Using the requirement that the mass of the gravitons must be positive (and real), we can fix the sign of the couplings in the NCG spectral action approach. We then calculate the energy loss for a circular binary system and compare it to the results obtained from standard General Relativity. We conclude that the amplitude of modifications within NCG is small, nevertheless the NCG approach leads to some distinctive features which we analyze. We round up with our conclusions in Section D

\section{NONCOMMUTATIVE GEOMETRY SPECTRAL ACTION}

In the NonCommutative Geometry [1, 2] approach, the Standard Model (SM) of electroweak and strong interactions is considered as a phenomenological model, which dictates the geometry of space-time, so that the associated Maxwell-Dirac action functional produces the SM with all known experimental results. The outcome of this approach is a geometric space defined by the product, $\mathcal{M} \times \mathcal{F}$, of a continuum compact Riemaniann manifold, $\mathcal{M}$, and a tiny discrete finite noncommutative space, $\mathcal{F}$, composed of only two points. Such an almost commutative space is the simplest extension of the more familiar commutative space upon which General Relativity is formulated. Certainly one should not expect the validity 
of this simplistic approach to hold at the Planck scale, which is the scale at which all notion of classical geometry loses its meaning.

The metric dimension of the product geometry $\mathcal{M} \times$ $\mathcal{F}$ is 4 , the same as the ordinary space-time manifold. Thus, the metric dimension of the noncommutative space $\mathcal{F}$ is zero, while for noncommutative spaces one must distinguish between the metric dimension and the $\mathrm{KO}$ dimension. The internal space $\mathcal{F}$ has $K O$-dimension 6 to allow fermions to be simultaneously Weyl and chiral, whilst it is discrete to avoid the infinite tower of massive particles that are produced in string theory.

The noncommutative nature of $\mathcal{F}$ is given by the real spectral triple $(\mathcal{A}, \mathcal{H}, D)$ that generalizes Riemannian geometry to the noncommutative setting; $\mathcal{A}$ is an involution of operators on the finite-dimensional Hilbert space $\mathcal{H}$ of Euclidean fermions, and $D$ is a self-adjoint unbounded operator in $\mathcal{H}$. The choice of Hilbert space has no importance, since all separable infinite-dimensional Hilbert spaces are isomorphic. The algebra $\mathcal{A}$, related to the gauge group of local gauge transformations, is the algebra of coordinates. A space is described by the algebra of coordinates, which in the context of NCG is represented as operators on a Hilbert space. Since real coordinates are represented by self-adjoint operators, all information about a space within NCG is encoded in the algebra of coordinates $\mathcal{A}$. By assuming that the algebra constructed in $\mathcal{M} \times \mathcal{F}$ is symplectic-unitary, $\mathcal{A}$ must be of the form

$$
\mathcal{A}=M_{a}(\mathbb{H}) \oplus M_{k}(\mathbb{C}) ;
$$

$k=2 a, \mathbb{H}$ is the algebra of quaternions. The choice $k=4$ is the first value that produces the correct number of fermions in each generation, i.e., $k^{2}=16$ fermions in each of the three generations [3].

The operator $D$ corresponds to the inverse of the Euclidean propagator of fermions, and is given by the Yukawa coupling matrix which encodes the masses of the elementary fermions and the Kobayashi-Maskawa mixing parameters. The commutator $[D, a]$, with $a \in \mathcal{A}$, plays the rôle of the differential quotient $d a / d s$, with $d s$ the unit of length. The familiar geodesic formula

$$
d(x, y)=\inf \int_{\gamma} d s
$$

where the infimum is taken over all possible paths connecting $x$ to $y$, which is used to determine the distance $d(x, y)$ between two points $x$ and $y$ within Riemannian geometry, is replaced by

$$
d(x, y)=\sup \{|f(x)-f(y)|: f \in \mathcal{A},\|[D, f]\| \leq 1\},
$$

where $D$ is the inverse of the line element $d s$, within the noncommutative spectral geometry.

The fermions of the SM provide the Hilbert space $\mathcal{H}$ of a spectral triple for the algebra $\mathcal{A}$, while the bosons of the SM, including the Higgs boson, are obtained through inner fluctuations of the Dirac operator of the product
$\mathcal{M} \times \mathcal{F}$ geometry. Hence, the Higgs boson, which generates the masses of elementary particles through spontaneous symmetry breaking, becomes just a gauge field corresponding to a finite difference. Note that the corresponding mass scale specifies the inverse size of the discrete geometry $\mathcal{F}$.

Applying the spectral action principle, according to which the action functional on spectral triples depends only on the spectrum of the line element, i.e., the inverse of the Dirac operator, to the inner fluctuations of the product geometry $\mathcal{M} \times \mathcal{F}$, one recovers the SM coupled to gravity in the Euclidean form. Thus, the NCG spectral action approach - limited to the classical level even though it can a priori be quantized - offers an elegant geometric interpretation of the SM , the most successful phenomenological model of particle physics.

To be more precise, the SM Lagrangian - including mixing and Majorana mass terms for neutrinos, minimally coupled to gravity - can be successfully recovered from the asymptotic expansion of the spectral action functional

$$
\operatorname{Tr}\left(f\left(\frac{D}{\Lambda}\right)\right)
$$

where $f$ is a positive even function of the real variable and $\Lambda$ fixes the energy scale. Note that $D / \Lambda$ is dimensionless since the Dirac operator, being a differential operator, has dimensions of mass. The physical Lagrangian is thus obtained from the asymptotic expansion in the energy scale $\Lambda$ of the spectral action functional, Eq. (4). More precisely, using heat kernel methods one can write the square of the Dirac operator in terms of the inverse metric, the unit matrix and two matrix functions computed from $D$ and show that the trace, Eq. (4) above, can be expanded in a power series as a function of the inverse scale $\Lambda$ and it can thus be written in terms of the geometrical Seeley-deWitt coefficients $a_{n}$, as [11]

$$
\sum_{n=0}^{\infty} F_{4-n} \Lambda^{4-n} a_{n}
$$

where the function $F$ is defined such that $F\left(D^{2}\right)=f(D)$. Defining the moments

$$
f_{k}=\int_{0}^{\infty} f(u) u^{k-1} \mathrm{~d} u, \text { for } k>0,
$$

and $f_{0}=f(0)$, one finds

$$
\begin{aligned}
F_{4} & =2 f_{4} \\
F_{2} & =2 f_{2} \\
F_{0} & =f_{0} \\
F_{-2 n} & =\left[(-1)^{n}\left(\frac{\mathrm{d}}{2 u \mathrm{~d} u}\right)^{n} f\right](0) \text { for } n \geq 1,
\end{aligned}
$$

while the coefficients $a_{n}$ are known for any second order elliptic differential operator. 
The coupling with fermions can be obtained by including an additional fermionic term

$$
\frac{1}{2}<J \psi, D \psi>
$$

in Eq. (4), where $J$ is the real structure on the spectral triple and $\psi$ is a spinor in the Hilbert space $\mathcal{H}$ of the quarks and leptons.

The spectral action approach leads naturally to the merging of the three coupling constants at the unification scale, $g_{2}=g_{3}=\sqrt{5 / 3} g_{1}$, it provides neutrino masses and mixing as well as the see-saw mechanism, and it predicts a heavy Higgs mass.

The spectral action, Eq. (44), can be expanded in powers of the scale $\Lambda$ in the form

$\operatorname{Tr}\left(f\left(\frac{D}{\Lambda}\right)\right) \sim \sum_{k \in \operatorname{DimSp}} f_{k} \Lambda^{k} f|D|^{-k}+f(0) \zeta_{D}(0)+\mathcal{O}(1)$

where $f_{k}$ are the momenta of the function $f$ given in Eq. (6), the noncommutative integration is defined in terms of residues of zeta functions, and the sum is over points in the dimension spectrum of the spectral triple.

The physical Lagrangian that one obtains in this approach, contains, in addition to the full SM Lagrangian, the Einstein-Hilbert action with a cosmological term, a topological term related to the Euler characteristic of the space-time manifold, a conformal Weyl term and a conformal coupling of the Higgs field to gravity. Note that the coefficients of the gravitational terms depend on the Yukawa parameters of the particle physics content. Within the NCG spectral action, one works in Euclidean rather than Lorentzian signature, assuming that one can get back to the Minkowski signature through Wick rotation.

One then sets the parameters of the NCG spectral action at the (unification) scale $\Lambda$; predictions at lower energies are recovered by running the parameters down through Renormalization Group Equations (RGE). Hence, the spectral action at the unification scale $\Lambda$ offers a framework to investigate early universe cosmological models [6-10], while extrapolations to lower energies can be obtained via, firstly, RGE and secondly, inclusion of nonperturbative effects in the spectral action.

Adopting Euclidean signature, the gravitational part of the asymptotic formula for the bosonic sector of the NCG spectral action, including the coupling between the Higgs field $\phi$ and the Ricci curvature scalar $R$, is [4]

$$
\begin{array}{r}
\mathcal{S}_{\text {grav }}^{\mathrm{E}}=\int\left(\frac{1}{16 \pi G} R+\alpha_{0} C_{\mu \nu \rho \sigma} C^{\mu \nu \rho \sigma}+\tau_{0} R^{\star} R^{\star}\right. \\
\left.-\xi_{0} R|\mathbf{H}|^{2}\right) \sqrt{g} \mathrm{~d}^{4} x .
\end{array}
$$

Note that $\mathbf{H}$ is a rescaling $\mathbf{H}=\left(\sqrt{a f_{0}} / \pi\right) \phi$ of the Higgs field $\phi$ to normalize the kinetic energy; the momentum $f_{0}$ is physically related to the coupling constants at unification and the coefficient $a$ is related to the fermion and lepton masses and lepton mixing.

In the above action, Eq. (10), the first two terms only depend upon the Riemann curvature tensor; the first is the Einstein-Hilbert term with the second one being the Weyl curvature term. The third term

$$
R^{\star} R^{\star}=\frac{1}{4} \epsilon^{\mu \nu \rho \sigma} \epsilon_{\alpha \beta \gamma \delta} R_{\mu \nu}^{\alpha \beta} R_{\rho \sigma}^{\gamma \delta},
$$

is the topological term that integrates to the Euler characteristic and hence is nondynamical. The spectral action contains one more term that couples gravity with the SM, namely the last term in Eq. (10), which should always be present when one considers gravity coupled to scalar fields. This coupling can have significant consequences at high energies, such as in the early universe [6 10], however in this paper we will be concerned with the low energy weak curvature regime where this term is small.

Neglecting the nonminimal coupling between the Higgs field and the Ricci curvature, the equations of motion derived from the Lorentzian version of spectral action above read [6]

$$
\begin{array}{r}
R^{\mu \nu}-\frac{1}{2} g^{\mu \nu} R-32 \pi G \alpha_{0}\left[2 C_{; \lambda ; \kappa}^{\mu \lambda \nu \kappa}+C^{\mu \lambda \nu \kappa} R_{\lambda \kappa}\right] \\
=8 \pi G T_{\text {matter }}^{\mu \nu}
\end{array}
$$

implying that the NCG corrections vanish [6] for Friedmann-Lemaître-Robertson-Walker (FLRW) cosmologies. [The reader is directed to subsection IIIA for a definition and discussion of the Lorentzian conventions used.]

We will be concerned with linear perturbations around a Minkowski background metric in the synchronous gauge, so that the perturbed metric reads

$$
g_{\mu \nu}=\operatorname{diag}\left(\{a(t)\}^{2}\left[-1,\left(\delta_{i j}+h_{i j}(x)\right)\right]\right),
$$

where $a(t)$ is the cosmological scale factor. Throughout this paper we work in a flat background and hence $a(t)=$ 1 and $\dot{a} \equiv \mathrm{d} t / \mathrm{d} t=0$. The remaining gauge freedom can be completely fixed by setting $\nabla_{i} h^{i j}=0$. [A detailed discussion of this gauge fixing is given in subsection IIIC below.]

In Section III below we show that, the linearized equations of motion derived from the NCG spectral action, for such perturbations, read

$$
\left(\square-\beta^{2}\right) \square h^{\mu \nu}=\beta^{2} \frac{16 \pi G}{c^{4}} T_{\text {matter }}^{\mu \nu},
$$

where $T_{\text {matter }}^{\mu \nu}$ is taken to lowest order in $h^{\mu \nu}$. This implies that it is independent of $h^{\mu \nu}$ and satisfies the conservation equations

$$
\frac{\partial}{\partial x^{\mu}} T_{\nu}^{\mu}=0
$$


It is important to note that $\beta$, defined as

$$
\beta^{2} \equiv-1 /\left(32 \pi G \alpha_{0}\right)
$$

in Eq. (13), turns out to play the rôle of a mass and hence has to be real and positive, implying that $\alpha_{0}<0$. In the following we will see that, for $\alpha_{0}>0$, the gravitational waves evolve according to a Klein-Gordon like equation with a tachyonic mass, and hence the background, which in our case is Minkowski space, is unstable. We can thus restrict to $\alpha_{0}<0$ for Minkowski space to be a (stable) vacuum of the theory.

\section{PERTURBATION EQUATIONS}

To write down the linearized equations of motion, we will first discuss our conventions for the metric signature and the Ricci tensor.

\section{A. Conventions for $R_{\mu \nu}$ and Signature}

In this paper, we are using conventions in which the signature is $(-,+,+,+)$ and the Ricci tensor is defined as $R_{\mu \nu}=R_{\mu \nu \rho}^{\rho}$, with $R_{\mu \nu \rho}{ }^{\sigma} \omega_{\sigma}=\left[\nabla_{\mu}, \nabla_{\nu}\right] \omega_{\rho}$. In General Relativity such choices are merely conventions, which are relatively unimportant (provided of course that one is consistent), here however the situation is very different. The Lorentzian version of the NCG action, Eq. (10), that we use reads

$$
\begin{array}{r}
\mathcal{S}_{\text {grav }}^{\mathrm{L}}=\int\left(\frac{1}{16 \pi G} R+\alpha_{0} C_{\mu \nu \rho \sigma} C^{\mu \nu \rho \sigma}+\tau_{0} R^{\star} R^{\star}\right. \\
\left.-\xi_{0} R|\mathbf{H}|^{2}\right) \sqrt{-g} \mathrm{~d}^{4} x .
\end{array}
$$

It is thus clear that the conventions used to define, for example, $R_{\mu \nu}$ will radically alter the theory, unless one also alters the (signs) of the couplings. Specifically, consider using the opposite convention for the Ricci tensor, which introduces a negative sign on all terms depending on $R_{\mu \nu}$, but not on terms depending on $R_{\mu \nu \rho \sigma}$. Since our action now contains terms of both kinds (i.e., $R$ and $C_{\mu \nu \rho \sigma} C^{\mu \nu \rho \sigma}$ ), this change of convention introduces a relative sign change. This can simply be compensated for by changing $\alpha_{0} \rightarrow-\alpha_{0}$. However, without this change the action is very different. An exactly similar change happens if we considered a different choice of convention for the signature or the sign of $R_{\mu \nu \rho \sigma}$, as these both introduce a sign change in the $R$ term, but not the $C_{\mu \nu \rho \sigma} C^{\mu \nu \rho \sigma}$ term.

As shown in Refs. 6 9], the presence of the nonminimal coupling of curvature to the Higgs field can have significant effects of the cosmological dynamics and one may wonder whether the sign ambiguities discussed here may affect these results. Fortunately, from Eq. (16) it is clear that the relative sign between the Einstein-Hilbert term $(R)$ and the nonminimal coupling $\left(R|\mathbf{H}|^{2}\right)$ is inde- pendent of any convention (since they both contain $R$ ). In a cosmological setting (i.e., for FLRW geometries) the Weyl term vanishes and hence, in homogeneous cosmologies, the only NCG affects come from the nonminimal coupling [6], allowing such issues to be avoided.

Since the underlying NCG theory is only developed for the Euclidean signature, it does not provide a guide for the Wick rotation to the Lorentzian space. Hence the choice of the sign of the couplings, appropriate for a particular choice of convention, can only be made by testing the physical consequences of the theory. In the following section we will show that gravitational waves offer an excellent probe of the couplings in this theory, but even without an in-depth analysis, from Eq. (13) one can immediately see that the coupling $\alpha_{0}$ must be negative (in the conventions used here). If it were not, the $\beta$ parameter would be complex and this would correspond to a tachyonic mode of the graviton (we refer the reader to a discussion below). This would indicate that Minkowski space-time is unstable to small perturbations. If we rule out such a possibility on physical grounds (or require that the Lorentzian version of the NCG action should admit Minkowski as a stable vacuum) then we can restrict $\alpha_{0}<0$ with the conventions used here. Consider for example the consequence of changing the signature, so that $\square \rightarrow-\square$. Such a change of convention essentially changes the sign of $\beta^{2}$ and the conclusions would be reversed ${ }^{1}$.

\section{B. Linearized Equations of Motion}

Variation of the gravitational part action Eq. (16) w.r.t. the metric $g_{\mu \nu}$ leads to the following addition to the Einstein tensor of General Relativity (GR),

$$
\begin{aligned}
G_{\mathrm{NCG}}^{\mu \nu}= & -\frac{1}{2 \kappa} G_{\text {Einstein }}^{\mu \nu} \\
& +2 \alpha_{0}\left(2 \nabla \lambda \nabla \kappa C^{\mu \kappa \nu \lambda}+C^{\mu \kappa \nu \lambda} R_{\kappa \lambda}\right),
\end{aligned}
$$

where as usual

$$
G_{\text {Einstein }}^{\mu \nu}=R^{\mu \nu}-\frac{1}{2} g^{\mu \nu} R
$$

Given the convention used here to define the Ricci tensor the Weyl Tensor is explicitly given as,

$$
\begin{gathered}
C_{\mu \lambda \nu \kappa}=R_{\mu \lambda \nu \kappa}+\left(g_{\mu[\nu} R_{\kappa] \lambda}-g_{\lambda[\nu} R_{\kappa] \mu}\right) \\
-\frac{1}{3} g_{\mu[\nu} g_{\kappa] \lambda} R .
\end{gathered}
$$

\footnotetext{
1 The concerned reader should note that exactly the same situation arises in standard Klein-Gordon equation, where the sign of the mass term is changed under a change of signature.
} 
Using the contracted Bianchi identity

$$
\nabla^{\kappa} R_{\mu \lambda \nu \kappa}=-\left(\nabla_{\lambda} R_{\mu \nu}-\nabla_{\mu} R_{\lambda \nu}\right),
$$

and its remaining trace

$$
\nabla^{\kappa} R_{\lambda \kappa}=\frac{1}{2} \nabla_{\lambda} R
$$

we can arrive at the following expression

$$
\begin{aligned}
2 \nabla^{\lambda} \nabla^{\kappa} C_{\mu \lambda \nu \kappa}= & -C_{\lambda \mu \kappa \nu} R^{\lambda \kappa}-\nabla^{\lambda} \nabla \lambda\left(R_{\mu \nu}-\frac{1}{6} g_{\mu \nu} R\right) \\
& +\frac{1}{3} \nabla \mu \nabla \nu R-2 R_{\mu \rho} R^{\rho}{ }_{\nu}+\frac{2}{3} R R_{\nu \mu} \\
& +\frac{1}{2} g_{\mu \nu}\left(R_{\kappa \lambda} R^{\lambda \kappa}-\frac{1}{3} R^{2}\right) .
\end{aligned}
$$

Notice that the expression above for $2 \nabla \lambda \nabla{ }_{\kappa} C^{\mu \lambda \nu \kappa}$ shows that the $C^{\mu \kappa \nu \lambda} R_{\kappa \lambda}$ term in Eq. (17) exactly cancels in favor of terms which are of second order of solely the Ricci tensor and/or Ricci scalar.

We now follow the standard procedure of perturbing about a flat metric, where

$$
g_{\mu \nu}=\eta_{\mu \nu}+\gamma_{\mu \nu}, \quad g^{\mu \nu}=\eta^{\mu \nu}-\gamma^{\mu \nu}
$$

and

$$
\gamma=\gamma_{\mu}^{\mu}=\eta^{\mu \nu} \gamma_{\mu \nu}
$$

all tensor indices are raised and lowered using the background metric $\eta_{\mu \nu}$ (except for the indices of $g_{\mu \nu}$ and $g^{\mu \nu}$ ). To first order in metric perturbations we then have

$$
\begin{aligned}
& 2\left\{\nabla \lambda \nabla \kappa C^{\mu \kappa \nu \lambda}\right\}= \\
& \partial_{\lambda} \partial^{\lambda}\left(\partial^{\kappa} \partial^{(\nu} \bar{\gamma}^{\mu)}{ }_{\kappa}-\frac{1}{2} \partial_{\kappa} \partial^{\kappa} \bar{\gamma}^{\mu \nu}-\frac{1}{6} \eta^{\mu \nu} \partial^{\sigma} \partial^{\kappa} \bar{\gamma}_{\sigma \kappa}\right) \\
& -\partial_{\lambda} \partial^{\nu}\left(\partial^{\kappa} \partial^{(\lambda} \bar{\gamma}^{\mu)}{ }_{\kappa}-\frac{1}{2} \partial_{\kappa} \partial^{\kappa} \bar{\gamma}^{\lambda \mu}\right)+\frac{1}{6} \partial^{\mu} \partial^{\nu} \partial^{\lambda} \partial^{\kappa} \bar{\gamma}_{\lambda \kappa} \\
& -\frac{1}{6}\left(\eta^{\mu \nu} \partial_{\kappa} \partial^{\kappa}-\partial^{\mu} \partial^{\nu}\right) \partial_{\lambda} \partial^{\lambda} \gamma+\mathcal{O}\left(\gamma^{2}\right) .
\end{aligned}
$$

where $\mathcal{O}\left(\gamma^{2}\right)$ denotes any second order combinations of $\gamma_{\mu \nu}$ and we have defined

$$
\bar{\gamma}_{\mu \nu}=\gamma_{\mu \nu}-\frac{1}{2} \eta_{\mu \nu} \gamma,
$$

i.e., the trace reverse of $\gamma_{\mu \nu}$.

Similarly, to linear order in metric perturbations the Einstein tensor is simply

$$
G_{\text {Einstein }}^{\mu \nu}=+\frac{1}{2} \partial_{\lambda} \partial^{\lambda} \bar{\gamma}^{\mu \nu}+\mathcal{O}\left(\gamma^{2}\right)
$$

\section{Gauge Conditions}

In calculating [6] the linearized equations of motion, the traceless transverse gauge was imposed on the metric perturbations $h_{\mu \nu}$; here we explicitly show that this is indeed a valid choice. As before, we denote metric perturbations that have not been gauge fixed by $\gamma_{\mu \nu}$ and reserve $h_{\mu \nu}$ for the final, gauge fixed perturbations that correspond to the physical gravitational waves.

As always we have a freedom due to diffeomorphism invariance of the action to restrict the gauge of the metric perturbations. Explicitly, under a diffeomorphism generated by $\xi_{\mu}$ the metric perturbations $\gamma_{\mu \nu}$ transform as

$$
\gamma_{\mu \nu}^{\text {old }} \stackrel{\xi_{\mu}}{\longrightarrow} \gamma_{\mu \nu}^{\text {new }}=\gamma_{\mu \nu}^{\text {old }}+\partial_{\mu} \xi_{\nu}+\partial_{\nu} \xi_{\mu} .
$$

Without loss of generality, one can impose the Lorentz gauge conditions

$$
\partial^{\mu} \bar{\gamma}_{\mu \nu}=0
$$

restricting the perturbations to be transverse, where we introduced the "trace reverse" of $\gamma_{\mu \nu}$, as in Eq. (25).

Choosing this gauge (and dropping the label new on $\gamma_{\mu \nu}$ ), Eq. (24) simplifies to

$$
\begin{array}{r}
2\left\{\nabla \lambda \nabla_{\kappa} C^{\mu \lambda \nu \kappa}\right\}= \\
-\frac{1}{2} \partial_{\kappa} \partial^{\kappa}\left(\partial_{\lambda} \partial^{\lambda} \bar{\gamma}^{\mu \nu}+\frac{1}{3}\left(\eta^{\mu \nu} \partial_{\lambda} \partial^{\lambda}-\partial^{\mu} \partial^{\nu}\right) \gamma\right) .
\end{array}
$$

Combining the above result with the Einstein contribution, $G_{\text {Einstein }}^{\mu \nu}$, to the equation of motion, the left-hand side of Eq. (11) is, to first order in $\gamma_{\mu \nu}$, given by

$$
\begin{gathered}
G_{\text {NCG }}^{\mu \nu}=-G_{\text {Einstein }}^{\mu \nu}-\frac{1}{\beta^{2}}\left\{2 \nabla \kappa \nabla \lambda C^{\mu \lambda \nu \kappa}\right\} \\
=-\frac{1}{2} \partial_{\kappa} \partial^{\kappa} \bar{\gamma}^{\mu \nu} \\
+\frac{1}{2 \beta^{2}} \partial_{\kappa} \partial^{\kappa}\left(\partial_{\lambda} \partial^{\lambda} \bar{\gamma}^{\mu \nu}+\frac{1}{3}\left(\eta^{\mu \nu} \partial_{\lambda} \partial^{\lambda}-\partial^{\mu} \partial^{\nu}\right) \gamma\right) .
\end{gathered}
$$

However, the Lorentz gauge does not uniquely fix all the gauge freedom. More precisely, we still are free to perform gauge transformations, generated by any $\xi_{\mu}$, that satisfy

$$
\partial_{\mu} \partial^{\mu} \xi_{\nu}=0,
$$

since this still preserves the gauge condition, Eq. (28), as can be checked directly.

We can use this transformation, Eq. (31), to set (in the new frame) $\gamma=0$ and $\gamma_{0 i}=0(i=1,2,3)$ by solving the corresponding equations for $\xi_{i}$ and their time derivatives on some initial surface $t=t_{0}$ where no sources are present and further extending into a source free region $\left(\right.$ i.e., $\left.T^{\mu \nu}=0\right)$ [12]. After performing these gauge transformations the source free equations of motion (i.e., the 
left-hand side of Eq. (11)) read

$$
-\frac{1}{2} \partial_{\kappa} \partial^{\kappa} \bar{\gamma}_{\mu \nu}+\frac{1}{2 \beta^{2}} \partial_{\kappa} \partial^{\kappa} \partial_{\lambda} \partial^{\lambda} \bar{\gamma}_{\mu \nu}=0,
$$

where we have made repeated use of the fact that $\partial_{\mu} \partial^{\mu} \xi_{\nu}=0$.

Up to this point, the gauge restrictions $\gamma=0$ and $\gamma_{0 i}=0(i=1,2,3)$ are the same as those typically used in General Relativity, which is to be expected since all we have used is the diffeomorphism invariance of the action. However, in determining whether we can set $\gamma_{00}=0$, the equations of motion are used and hence one might expect that this gauge condition will be different than that of General Relativity. To confirm its validity note that Eq. (28) implies

$$
\frac{\partial \gamma_{00}}{\partial t}=0
$$

Now, using the equation of motion in the presence of matter source we arrive at

$$
\begin{aligned}
\nabla^{2} \gamma_{00}-\frac{1}{\beta^{2}} \nabla^{2}\left(\nabla^{2} \gamma_{00}\right) & =-\frac{2 \kappa}{c^{4}} T_{00} \\
\left(1-\frac{1}{\beta^{2}} \nabla^{2}\right) \nabla^{2} \gamma_{00} & =-\frac{2 \kappa}{c^{4}} T_{00} .
\end{aligned}
$$

Recall that General Relativity is recovered in this setting by taking $\beta \rightarrow \infty$. Thus, one can see that in this limit the equation simplifies to $\nabla^{2} \gamma_{00}=-(16 \pi G) / c^{4} T_{00}$, which fixes $\gamma_{00}$ to be a constant (assuming the spacetime is asymptotically flat) away from the source. Finally, a redefinition (gauge transformation) allows us to set $\gamma_{00}=0$.

From Eq. (34) we see that, away from the source, $\nabla^{2} \gamma_{00}=0$ is still a solution and hence we can fix $\gamma_{00}=0$, however this is no longer the only solution to Eq. (34). In particular, away from the source one could fix $\gamma_{00}$ via,

$$
\left(1-\frac{1}{\beta^{2}} \nabla^{2}\right) \gamma_{00}=0,
$$

which clearly solves Eq. (34). This would result in a modification of what is often referred to as the radiation gauge. In the following, we choose $\gamma_{00}=0$ so as to be able to directly compare our results to the standard ones obtained within General Relativity.

In cases where sources are present, the NCG equation of motion with gravity and normal matter is

$$
\begin{aligned}
& \partial_{\kappa} \partial^{\kappa} \bar{\gamma}^{\mu \nu}-\frac{1}{\beta^{2}} \partial_{\kappa} \partial^{\kappa}\left[\partial_{\lambda} \partial^{\lambda} \bar{\gamma}^{\mu \nu}+\frac{1}{3}\left(\eta^{\mu \nu} \partial_{\lambda} \partial^{\lambda}-\partial^{\mu} \partial^{\nu}\right) \gamma\right] \\
& =-\frac{2 \kappa}{c^{4}} T^{\mu \nu} .
\end{aligned}
$$

Since $T_{\mu \nu} \neq 0$ we are not free to impose the traceless condition of the radiation gauge ${ }^{2}$. But the explicit presence of the trace $\gamma$ in Eq. (36) above can be eliminated by formally defining the tensor $\bar{h}_{\mu \nu}$ as,

$$
\bar{h}_{\mu \nu}=\bar{\gamma}_{\mu \nu}-\frac{1}{3 \beta^{2}} \mathcal{O}^{-1}\left(\eta_{\mu \nu} \square-\partial_{\mu} \partial_{\nu}\right) \gamma,
$$

where the operator $\mathcal{O}$ is given by

$$
\mathcal{O}=\left(1-\frac{\square}{\beta^{2}}\right) \text {. }
$$

This is a modification of the trace reverse of the metric perturbations that is usually used, however it performs the same task, namely removing the trace from the equations of motion, Eq. (36). As it can be easily checked, the Lorentz gauge condition, Eq. (28), is satisfied by $\bar{h}_{\mu \nu}$ as long as it is also satisfied by $\bar{\gamma}_{\mu \nu}$. Note that the trace of $\bar{h}_{\mu \nu}$ is

$$
\bar{h}=-\left(1+\frac{\mathcal{O}^{-1} \square}{\beta^{2}}\right) \gamma,
$$

so we see that indeed, this reproduces the trace reverse of $\gamma_{\mu \nu}$ in the limit $\beta \rightarrow \infty$. Clearly then, when we are away from a source, we can impose that $\gamma=0$ and this implies that $\bar{h}=0$.

In terms of $\bar{h}_{\mu \nu}$ the equation of motion, Eq. (36), is

$$
\left(1-\frac{1}{\beta^{2}} \square\right) \square \bar{h}^{\mu \nu}=-\frac{16 \pi G}{c^{4}} T^{\mu \nu} .
$$

Dropping the over-bars, this is exactly Eq. (13).

\section{Green's Function}

The general physical solution to Eq. (40) is given by

$$
h^{\mu \nu}=2 \beta^{2} \kappa \int \mathrm{d} S\left(x^{\prime}\right) G_{\mathrm{R}}\left(x, x^{\prime}\right) T^{\mu \nu}\left(x^{\prime}\right),
$$

where the Green's function $G_{\mathrm{R}}\left(x, x^{\prime}\right)$ satisfies the fourthorder partial differential equation:

$$
\left(\square-\beta^{2}\right) \square G_{\mathrm{R}}\left(x, x^{\prime}\right)=4 \pi \delta^{(4)}\left(x-x^{\prime}\right),
$$

where the operators $\square$ above are acting on $x$. In order to find a solution $G_{\mathrm{R}}\left(x, x^{\prime}\right)$ to Eq. (42) consider two distributions $g_{1}$ and $g_{2}$ which satisfy the following second-order equations:

$$
\begin{aligned}
\left(\square-\beta^{2}\right) g_{1} & =4 \pi \delta^{(4)}\left(x-x^{\prime}\right), \\
\square g_{2} & =4 \pi \delta^{(4)}\left(x-x^{\prime}\right) .
\end{aligned}
$$

\footnotetext{
2 Nor would this be possible if one had chosen the modified radiation gauge implied by Eq. 35.
} 
Then one can easily verify that the combination

$$
G_{\mathbf{R}}\left(x, x^{\prime}\right)=\frac{1}{\beta^{2}}\left(g_{1}-g_{2}\right),
$$

will be a solution to Eq. (42). Physically we are interested in the retarded Green's function solution to Eq. (42), that is of the form

$$
G_{\mathrm{R}}\left(x, x^{\prime}\right)=\Theta\left(t-t^{\prime}\right) g\left(x-x^{\prime}\right),
$$

where $\Theta(z)$ is the Heavyside step function. So $g_{1}$ and $g_{2}$ in Eq. (45) above must each be retarded solutions to Eqs. (43) and (44), respectively. The other three combinations of retarded and advanced solutions violate causality and will not be considered further. So we have reduced the problem of finding the retarded Green's function solution of the fourth-order, Eq. (42), to finding the retarded Green's function solutions of the two secondorder differential equations, Eqs. (43) and (44). The explicit calculations are given in the Appendix and result in:

$$
\begin{aligned}
& g_{1 \mathrm{R}}=\left\{\frac{\beta \mathcal{J}_{1}(\beta \tau)}{\tau} \Theta(c T-|\mathbf{R}|)-2 \delta\left(\tau^{2}\right)\right\} \Theta(T), \\
& g_{2 \mathrm{R}}=-2 \delta\left(\tau^{2}\right) \Theta(T)
\end{aligned}
$$

where we have defined

$$
\begin{aligned}
T & \equiv t-t^{\prime} \\
\mathbf{R} & \equiv \mathbf{r}-\mathbf{r}^{\prime} \\
\tau & \equiv \sqrt{(c T)^{2}-|\mathbf{R}|^{2}}
\end{aligned}
$$

and $\mathcal{J}_{1}(x)$ is the first order Bessel function of the first kind. Thus, Eq. (45) implies that

$$
G_{\mathrm{R}}\left(x, x^{\prime}\right)=\frac{\mathcal{J}_{1}(\beta \tau)}{\beta \tau} \Theta(c T-|\mathbf{R}|) \Theta(T) .
$$

Note the absence of the delta function singularities on the light cone, consistent with the general analysis detailed in Ref. [20].

Using Eq. (41) one finds that the field is given by

$$
\begin{array}{r}
h^{\mu \nu}(\mathbf{r}, t)=\frac{4 G \beta}{c^{4}} \int \mathrm{d} \mathbf{r}^{\prime} \mathrm{d} t^{\prime} \frac{\Theta(T)}{\sqrt{(c T)^{2}-|\mathbf{R}|^{2}}} \\
\times \mathcal{J}_{1}\left(\beta \sqrt{(c T)^{2}-|\mathbf{R}|^{2}}\right) T^{\mu \nu}\left(\mathbf{r}^{\prime}, t^{\prime}\right) \\
\times \Theta(c T-|\mathbf{R}|) .
\end{array}
$$

One thus finds that the field is sourced only by regions within our past light code (i.e., $c T>|\mathbf{R}|$ ), which is expected for the propagation of a (positive-real) massive field. Notice that if $\beta^{2}<0$, corresponding to $\alpha_{0}>0$, we find that an observed field is sourced from regions with space-like separation. This is due to the fact that $\beta^{2}<0$ corresponds to a tachyon (complex mass) mode of the gravitational wave.

If we consider the far-field limit, i.e. $|\mathbf{r}| \approx\left|\mathbf{r}-\mathbf{r}^{\prime}\right|$, we can write the spatial components of this field as

$$
\begin{aligned}
h^{i k}(\mathbf{r}, t) & \approx \frac{2 G \beta}{3 c^{4}} \int_{-\infty}^{t-\frac{1}{c}|\mathbf{r}|} \frac{\mathrm{d} t^{\prime}}{\sqrt{c^{2}\left(t-t^{\prime}\right)^{2}-|\mathbf{r}|^{2}}} \\
& \times \mathcal{J}_{1}\left(\beta \sqrt{c^{2}\left(t-t^{\prime}\right)^{2}-|\mathbf{r}|^{2}}\right) \ddot{D}^{i k}\left(t^{\prime}\right)
\end{aligned}
$$

where we have, as usual, introduced the quadrupole moment,

$$
D^{i k}(t) \equiv \frac{3}{c^{2}} \int \mathrm{d} \mathbf{r} x^{i} x^{k} T^{00}(\mathbf{r}, t),
$$

the second time derivative of which is given by

$$
\int \mathrm{d} \mathbf{r} T^{i k}(\mathbf{r}, t)=-\frac{1}{6} \frac{\mathrm{d}^{2}}{\mathrm{~d} t^{2}}\left[D^{i k}(t)\right] .
$$

In conclusion, just as in the case of General Relativity, we find that the gravitational waves are only sourced from systems with a nontrivial quadrupole moment. This is essentially due to the conservation of the energy momentum tensor, which is unaltered in this theory. However, the propagation of gravitational waves is significantly altered, by the presence of additional massive modes.

\section{EXAMPLES}

As a simple pedagogical example let us consider the case of $\ddot{D}^{i k} \approx$ constant, for which one can explicitly perform the integral in Eq. (51) to find

$$
\begin{aligned}
h^{i k}(\mathbf{r}, t) \approx & -\left.\frac{2 G}{3 c^{2}|\mathbf{r}|} \ddot{D}^{i k}\right|_{\text {fixed }} \\
& \times[\sinh (\beta|\mathbf{r}|)-\cosh (\beta|\mathbf{r}|)+1] .
\end{aligned}
$$

Thus, one recovers the standard result in the $\beta \rightarrow \infty$ limit, namely

$$
\begin{aligned}
\lim _{\beta \rightarrow \infty} h^{i k}(\mathbf{r}, t) & =\lim _{\beta \rightarrow \infty}\left[-\left.\frac{2 G}{3 c^{4}|\mathbf{r}|} \ddot{D}^{i k}\right|_{\text {fixed }}\left(1-e^{-\beta|\mathbf{r}|}\right)\right] \\
& ={ }^{(\mathrm{GR})} h^{i k}(\mathbf{r}, t) \lim _{\beta \rightarrow \infty}\left(1-e^{-\beta|\mathbf{r}|}\right),
\end{aligned}
$$

where ${ }^{(\mathrm{GR})} h^{i k}$ denotes the field in the General Relativistic case. This is expected, since the $\beta \rightarrow \infty$ limit corresponds to taking $\alpha_{0} \rightarrow 0$ and, as it can be seen from either Eq. (10) or Eq. (13), one then recovers the EinsteinHilbert result.

The next simplest example is a system with a periodically varying quadrupole moment, i.e.

$$
\ddot{D}^{i j}(t)=A^{i j} \cos \left(\omega^{i j} t+\phi^{i j}\right),
$$


where $A^{i j}$ is a constant in time, $\omega^{i j}$ is the frequency of the oscillations of the $i j$ component and $\phi^{i j}$ is the phase, both of which we consider to be time independent. No summation over $i, j$ is implied. Using this in Eq. (51) one finds

$$
\begin{aligned}
\dot{h}^{i j}= & \frac{4 G \beta A^{i j} \omega^{i j}}{3 c^{4}}\left[\sin \left(\omega^{i j} t+\phi^{i j}\right) f_{c}\left(\beta|\mathbf{r}|, \frac{\omega^{i j}}{\beta c}\right)\right. \\
& \left.+\cos \left(\omega^{i j} t+\phi^{i j}\right) f_{s}\left(\beta|\mathbf{r}|, \frac{\omega^{i j}}{\beta c}\right)\right],
\end{aligned}
$$

where again no summation is implied and we have defined the functions,

$$
\begin{aligned}
& f_{\mathrm{s}}(x, z) \equiv \int_{0}^{\infty} \frac{\mathrm{d} s}{\sqrt{s^{2}+x^{2}}} \mathcal{J}_{1}(s) \sin \left(z \sqrt{s^{2}+x^{2}}\right), \\
& f_{\mathrm{c}}(x, z) \equiv \int_{0}^{\infty} \frac{\mathrm{d} s}{\sqrt{s^{2}+x^{2}}} \mathcal{J}_{1}(s) \cos \left(z \sqrt{s^{2}+x^{2}}\right) .
\end{aligned}
$$

These functions are highly oscillatory, with somewhat different behavior for $z>1$ and $z<1$. Because they have a typical frequency of the order of $z$, which is (in general) different to $\omega^{i j}$, the wave-form of the gravitational radiation, Eq. (51) and its time derivative, Eq. (57) can experience beat phenomena. In particular, interference between the various functions can result in a significant enhancement of the amplitude.

As a specific example, of significant physical interest, consider a pair of masses $m_{1}$ and $m_{2}$, in a circular binary system, under the assumption that the internal structure of the bodies can be neglected. For such a system, orbiting in the $x y$-plane, one finds that the only nonzero components of the quadrupole are [17],

$$
\begin{aligned}
\ddot{D}^{x x}(t) & =12 \mu|\rho|^{2} \sin (2 \psi(t)) \omega^{3} \\
& =-\ddot{D}^{y y}(t), \\
\ddot{D}^{x y}(t) & =-12 \mu|\rho|^{2} \cos (2 \psi(t)) \omega^{3}, \\
D^{z z} & =-\mu|\rho|^{2},
\end{aligned}
$$

where $\mu=m_{1} m_{2} /\left(m_{1}+m_{2}\right)$ is the reduced mass of the system, $|\rho|$ is the magnitude of the separation vector between the bodies, which is constant for circular orbits, $\psi$ is the angle of the bodies relative to the $x$-axis and $\omega=\dot{\psi}$ is the orbital frequency, which for this simple system is a constant given by

$$
\omega \equiv \dot{\psi}=|\rho|^{-3 / 2} \sqrt{G\left(m_{1}+m_{2}\right)} .
$$

Using Eq. (51) one finds

$$
\begin{aligned}
\dot{h}^{i j} \dot{h}_{i j} & =\frac{9 \mu^{2}|\rho|^{2} \omega^{4} G^{2} \beta^{2}}{c^{6}} \\
& \times\left[f_{\mathrm{c}}^{2}\left(\beta|\mathbf{r}|, \frac{2 \omega}{\beta c}\right)+f_{\mathrm{s}}^{2}\left(\beta|\mathbf{r}|, \frac{2 \omega}{\beta c}\right)\right] .
\end{aligned}
$$

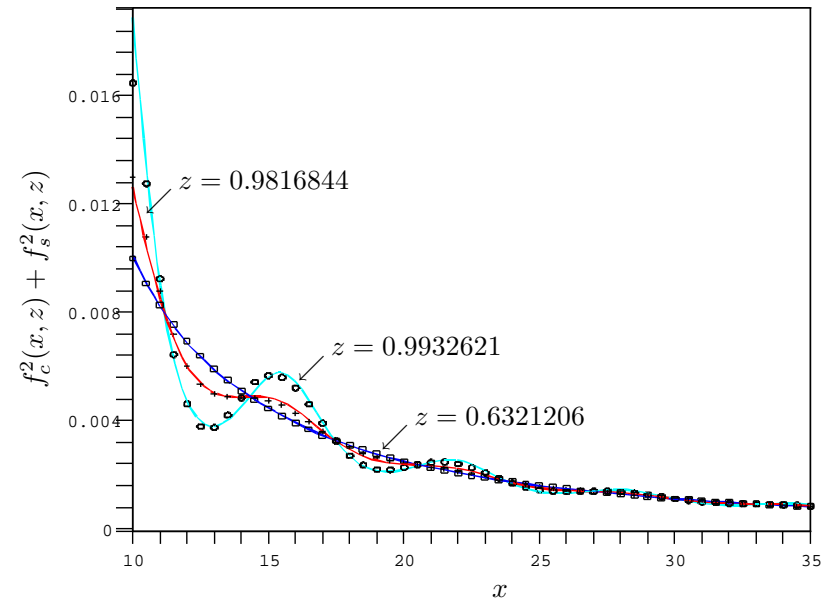

FIG. 1: The points are the numerical evaluation of $f_{\mathrm{c}}^{2}(x, z)+$ $f_{\mathrm{s}}^{2}(x, z)$ (for three different values of $z<1$ ) and the lines are plots of the corresponding fitted function given in Eq. (63). Notice that the fitting function breaks down as we approach $z \rightarrow 1$, which corresponds to $2 \omega \rightarrow \beta c$.

Following the standard approach (see e.g., Ref. [17]), one finds that the rate of energy loss from a system, in the far field limit, is given by

$$
-\frac{\mathrm{d} \mathcal{E}}{\mathrm{d} t} \approx \frac{c^{2}}{20 G}|\mathbf{r}|^{2} \dot{h}_{i j} \dot{h}^{i j} .
$$

This allows us to explicitly test the approximation against binary pulsar measurements, for which the energy loss has been very well characterized [18].

For a quantitative fit to the data one would have to extend this example to non-circular orbits and also account for tidal and other near field effects. However, such calculations rapidly become rather involved and even within this simple (and important) system one can derive some general consequences.

The functions in Eq. (58) are highly resonant at $z=$ 1 , which corresponds to an orbital frequency $\omega=\beta c / 2$, however they are readily calculated for both $z<1$ and $z>1$. In these regions the functions given in Eq. (58) can be evaluated numerically and fitted to an explicit functional forms. For $\omega<\beta c / 2$ this gives

$$
\begin{array}{r}
{\left[f_{\mathrm{c}}\left(\beta|\mathbf{r}|, \frac{2 \omega}{\beta c}\right)\right]^{2}+\left[f_{\mathrm{s}}\left(\beta|\mathbf{r}|, \frac{2 \omega}{\beta c}\right)\right]^{2}} \\
\approx \frac{1}{(\beta|\mathbf{r}|)^{2}} \exp \left(\frac{C}{\beta|\mathbf{r}|\left(1-\frac{2 \omega}{c \beta}\right)} \mathcal{J}_{1}\left(\beta|\mathbf{r}|-\frac{2 \omega}{c \beta}\right)\right),
\end{array}
$$

where $C \approx 0.175$ is approximately a constant except as $2 \omega \rightarrow \beta c$. In Fig. 1 we illustrate some examples of this approximation and show that the approximation is good even for $2 \omega / \beta c \approx 0.99$. 


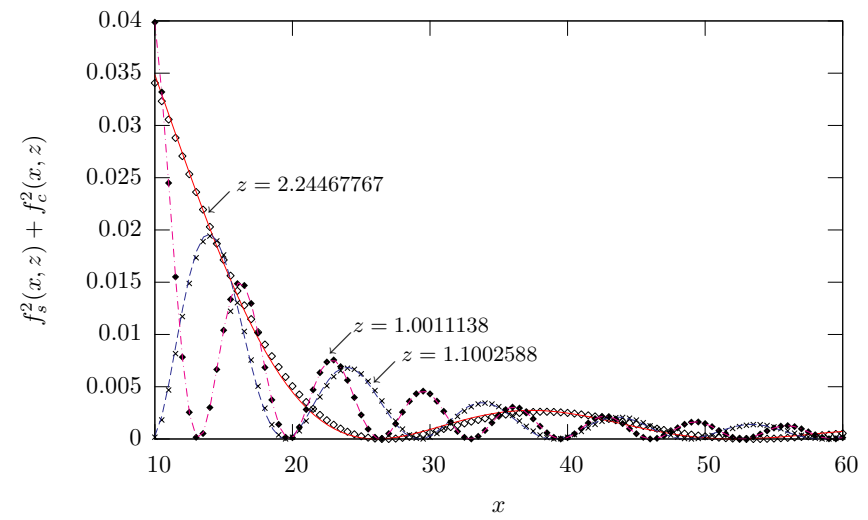

FIG. 2: The points are the numerical evaluation of $f_{\mathrm{c}}^{2}(x, z)+$ $f_{\mathrm{s}}^{2}(x, z)$ (for three different values of $z>1$ ) and the lines are plots of the corresponding fitted function given in Eq. (64). Notice that the fitting function remain a good approximation even as $z \rightarrow 1$, which corresponds to $2 \omega \rightarrow \beta c$.

Whilst for $\omega>\beta c / 2$ one finds

$$
\begin{aligned}
& {\left[f_{\mathrm{c}}\left(\beta|\mathbf{r}|, \frac{2 \omega}{\beta c}\right)\right]^{2}+\left[f_{\mathrm{s}}\left(\beta|\mathbf{r}|, \frac{2 \omega}{\beta c}\right)\right]^{2} } \\
\approx & \frac{4}{(\beta|\mathbf{r}|)^{2}} \sin ^{2}\left(\beta|\mathbf{r}|\left(\tilde{f}\left(\frac{2 \omega}{\beta c}\right)\right)^{-1}\right),
\end{aligned}
$$

where for the function $\tilde{f}$ is approximately

$$
\begin{aligned}
\tilde{f}\left(\frac{2 \omega}{\beta c}\right) \approx & 4 \sqrt{\left(\frac{2 \omega}{\beta c}\right)^{2}-1} \\
& +2 \exp \left(-\sqrt{\left(\frac{2 \omega}{\beta c}\right)^{2}-1}\right) .
\end{aligned}
$$

Figure 2 shows some examples of the quality of this fit, which, just as for Eq. (63), is best away from $\omega=\beta c / 2$, however remains very good even as one approaches this limit. It is important to note that Eq. (64) does not approximate the General Relativity solution for $\beta \rightarrow \infty$, since, in this limit, all (finite) orbital frequencies satisfy $\omega<\beta c / 2$.

Using the approximation given in Eq. (63), one can check that, for slow orbital frequencies, the expected result of General Relativity is indeed recovered in the $\beta \rightarrow \infty$ limit. Specifically, we can expand Eq. (62) in the large distance (large $|\mathbf{r}|)$ and small orbital frequency (i.e., $2 \omega \ll c \beta$ ) limit, to find the first order corrections to the standard result of General Relativity, namely

$$
\begin{aligned}
-\frac{\mathrm{d} \mathcal{E}}{\mathrm{d} t} & \approx \frac{32 G \mu^{2} \rho^{2} \omega^{6}}{5 c^{5}} \\
& \times\left[1+\frac{C}{\beta|\mathbf{r}|\left(1-\frac{2 \omega}{\beta c}\right)} \mathcal{J}_{1}\left(\beta|\mathbf{r}|-\frac{2 \omega}{\beta c}\right)+\ldots\right]
\end{aligned}
$$

Thus, the $\beta \rightarrow \infty$ (i.e., $\alpha_{0} \rightarrow 0$ ) limit reproduces the General Relativity result, as it should. Also note that any deviation from the standard result is suppressed by the distance to the source, at least for orbital frequencies small compared to $\beta c$. Although the amplitude of the deviation from the standard result is small, there are two interesting features: firstly, the existence of a maximum frequency $\beta c$ and secondly, the fact that the deviation is oscillatory.

The maximum frequency comes from the fact that, in addition to a natural speed $c$, this theory has a natural length, given by $\beta^{-1}=\sqrt{-\alpha_{0} G}$. This natural length scale comes from the first two moments of the test function used to define the spectral action, Eq. (7). Physically, one can think of this as the scale at which noncommutative effects become dominant. This is extremely suggestive of an underlying maximum frequency, which would have rather significant consequences for the particle physics sector of the theory, in particular for renormalization. However, it must be remembered that here we are working with one simple system and even in this case Eqs. (63)- 64) are numerical approximations. Nonetheless, the existence of a maximum frequency in this system allows NCG effects to significantly enhance the production of gravitational radiation. This is particularly important given the suppression with $1 /|\mathbf{r}|$, that is present in Eq. (66).

The presence of the Bessel function in Eq. (66) means that the amplitude of the deviation from the standard result of General Relativity will oscillate both with changing distances and changing frequencies. This allows for a myriad of possible observational signatures, such as distinct beats of the observed energy loss of binary pulsars, correlated to their changing orbital frequency and the distance to the binary. The fact that a similar phenomena occurs for the gravitational wave itself, Eq. (51), implies that there would also be a beat structure in direct detection observations. In the case of a binary pair the amplitude of the beat will be heavily suppressed compared to that of the carrier wave and thus it is likely to remain below observational sensitivity, except in systems with very large orbital frequency.

\section{CONCLUSIONS}

NonCommutative Geometry is a natural extension to our familiar notions of Riemannian geometry, that has the additional benefit of producing the action of all the Standard Model fields in addition to gravity terms, purely through geometrical considerations. Thus NCG treats both gravity and matter on an equal footing and provides us with concrete relationships between matter and gravitational couplings. The gravitational sector of (the asymptotic expansion of) this theory produces modifications to General Relativity and in this paper we explore the ramifications of these modifications on the formation and evolution of gravitational waves. 
We have shown that the theory contains both massive and massless gravitons and that the requirement that the mass of these gravitons be positive fixes the sign of one of the couplings in the theory (for a given choice of sign conventions). We also show that both these modes are sourced by the quadrupole moment of a system (just as in standard GR) and that the retarded Green's function is not restricted to the past light cone of the observer (unlike GR), as one would expect for a system with massive modes. We have explicitly calculated the energy loss for a circular binary system and compared the results to those of standard GR. We have demonstrated that the amplitude of these NCG modifications is suppressed by the distance between the observer and the source of the gravitational waves and hence will typically be small.

Despite the extremely small amplitude of deviations from standard results, we have shown that NCG produces several distinctive features. Firstly, the amplitude of the energy lost by a binary pair can be higher or lower than the expected value, depending on the orbital period of the pair and the distance to the observer. This opens up the possibility that the observed energy loss from such a pair would be seen to oscillate as the binary moves with respect to the Earth. Whilst such effects are likely to be beyond current observational resolution, they allow for an unexpected beat phenomenon, which would be a concrete signature of NCG.

In addition, we have shown that the amplitude of these effects is (approximately) proportional to $(1-2 \omega / c \beta)^{-1}$, where $\omega$ is the orbital frequency of the binary. Thus, it would appear that the NCG corrections to the energy loss by the binary can become arbitrarily large as the frequency of the binary approach the critical frequency $\beta c$. In such a regime, the weak field approach taken here would no longer be valid (and numerical approximations break down), so one would not trust systems very close to this limit, however it is certainly true that astrophysical constraints on the parameters of the theory will be significantly improved for objects with a very rapidly changing quadrupole moment. A precise understanding of such systems is likely to require detailed knowledge of various astrophysical effects (radiation and particle production, tidal stripping etc.) as well as analytic solutions to the graviton field in the large frequency regime.

Finally, the form of Eq. (57) suggests that similar behaviour may be present in other systems, with periodic, or almost periodic, variations in the (mass) quadrupole moment. For laboratory systems, the gravitational radiation predicted by General Relativity is negligible, however if the NCG enhancement were sufficiently large, this may no longer be true. Laboratory systems regularly have very large oscillation frequencies (e.g., lattice vibrations in solids can easily exceed $10^{12} \mathrm{~Hz}$ ) which would experience anomalous damping, if the system was producing significant amounts of gravitational radiation. This opens up the (remote) possibility that the noncommutative nature of space-time might be probed in the laboratory.

One can immediately use the results of this paper to examine circular binary systems, in order to constrain the value of $\beta$ [10]. Similarly, one can include eccentricity which may result in more restrictive constraints on the theory. An alternative avenue would be to use the gravitational wave-forms given here to deduce the consequences for direct gravity wave searches (LIGO, VIRGO, LISA, etc). In particular, to extend these result to the large field regime and look for modifications to the chirp that develops at the end of in-spiral events.

\section{Acknoweldgments}

The work of W. N. is supported in part by the NSF grant PHY0854743, the George A. and Margaret M. Downsbrough Endowment and the Eberly research funds of Penn State. The work of M. S. is partially supported by the European Union through the Marie Curie Research and Training Network UniverseNet (MRTN-CT2006-035863). J. O. acknowledges support from the Alfred P. Sloan Foundation and the Eberly College of Science.
[1] A. Connes, Noncommutative Geometry, Academic Press, New York (1994).

[2] A. Connes and M. Marcolli, Noncommutative Geometry, Quantum Fields and Motives, Hindustan Book Agency, India (2008).

[3] A. H. Chamseddine and A. Connes, arXiv:0706.3690 [hep-th]].

[4] A. H. Chamseddine, A. Connes and M. Marcolli, Adv. Theor. Math. Phys. 11, 991 (2007) arXiv:hep-th/0610241.

[5] T. v. d. Broek and W. D. van Suijlekom, arXiv:1003.3788 [hep-th].

[6] W. Nelson and M. Sakellariadou, Phys. Rev. D 81, 085038 (2010) arXiv:0812.1657 [hep-th]].
[7] W. Nelson and M. Sakellariadou, Phys. Lett. B 680, 263 (2009) arXiv:0903.1520 [hep-th]].

[8] M. Marcolli and E. Pierpaoli, arXiv:0908.3683 [hep-th].

[9] M. Buck, M. Fairbairn and M. Sakellariadou, arXiv:1005.1188 [hep-th]].

[10] W. Nelson, J. Ochoa and M. Sakellariadou, "Constraining the Noncommutative Spectral Action via Astrophysical Observations" (in prepapration).

[11] A. H. Chamseddine and A. Connes, J. Math. Phys. 47, 063504 (2006).

[12] R. M. Wald, General Relativity, University of Chicago Press, Chicago (1984).

[13] S. Deser, R. Jackiw and S. Templeton, Annals Phys. 140, 372 (1982); Erratum-ibid. 185 (1988) 406. 
[14] S. Deser, R. Jackiw and S. Templeton, Phys. Rev. Lett. 48, 975 (1982).

[15] D. Bessada and O. D. Miranda, Class. Quant. Grav. 26, 045005 (2009) arXiv:0901.1119 [gr-qc]].

[16] D. Bessada and O. D. Miranda, JCAP 0908, 033 (2009) arXiv:0908.1360 [astro-ph.CO]].

[17] L. D. Landau, E. M. Lifshitz, The classical theory of fields, Elsevier, Oxford (1975).

[18] C. M. Will, Liv. Rev. in Rel. 93 (2006).

[19] M. Kramer et al., Science 314, 97 (2006) arXiv:astro-ph/0609417.

[20] S. Prakash, Proc. Math. Sci. 31, 240 (1950).

[21] E. Poisson, Liv. Rev. Rel. 76 (2004).

[22] M. Abramowitz and I. A. Stegun, Handbook of Mathematical Functions with Formulas, Graphs, and Mathematical Tables, Dover Press, New York (1964).

\section{Appendix}

In what follows, we shall explicitly detail the calculations which were performed in order to determine the Green's functions, namely Eqs. (47), (48).

Consider first the following fourth-order wave equation

$$
\left(\square-\beta^{2}\right) \square G_{\mathrm{R}}\left(x, x^{\prime}\right)=4 \pi \delta^{(4)}\left(x-x^{\prime}\right) .
$$

Lorentz symmetry of the background restricts $G_{\mathrm{R}}$ to be solely a function of $x-x^{\prime}$. Given the following inverse Fourier transforms:

$$
G_{\mathrm{R}}\left(x, x^{\prime}\right)=\frac{1}{(2 \pi)^{4}} \int \mathrm{d}^{4} k \tilde{G}_{\mathrm{R}}(k) e^{i k \cdot\left(x-x^{\prime}\right)},
$$

and

$$
\delta\left(x-x^{\prime}\right)=\frac{1}{(2 \pi)^{4}} \int \mathrm{d}^{4} k e^{i k \cdot\left(x-x^{\prime}\right)},
$$

where $k \cdot z=-\omega z_{0}+\mathbf{k} \cdot \mathbf{z}$, the Fourier transform $\tilde{G}_{\mathrm{R}}(k)$ must satisfy

$$
\tilde{G}_{\mathrm{R}}(k)=\frac{4 \pi}{\left[(\omega+i \epsilon)^{2}-\mathbf{k}^{2}-\beta^{2}\right]\left[(\omega+i \epsilon)^{2}-\mathbf{k}^{2}\right]},
$$

in order to solve Eq. (A-1).

Upon performing the inverse Fourier transform to determine the coordinate expression for $G_{\mathrm{R}}\left(x, x^{\prime}\right)$, the following pole prescription uniquely determines the retarded Green's function (the reader is referred to Fig. 33). The contours are traversed above the poles in the complex $\omega$ plane: for $t-t^{\prime}>0$ we close the contour in the lower half plane picking up the residue of the poles; for $t-t^{\prime}<0$ we close the contour in the upper half plane, thus enclosing no poles.

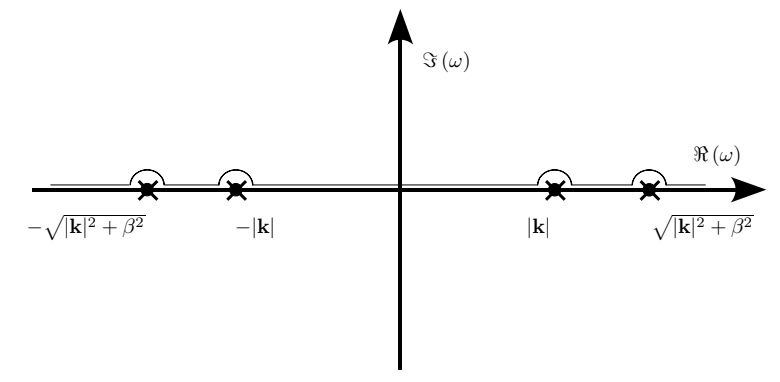

FIG. 3: The retarded Green's function is defined by extending the contour of the integral into the positive half of the imaginary plane around each of the four poles, $\omega= \pm|\mathbf{k}|$ and $\omega= \pm \sqrt{|\mathbf{k}|^{2}+\beta^{2}}$.

Note that $\tilde{G}_{\mathrm{R}}(k)$ can be rewritten as

$$
\begin{aligned}
\tilde{G}_{\mathrm{R}}(k)=4 \pi\left\{\frac{1}{\beta^{2}\left[(\omega+i \epsilon)^{2}-\mathbf{k}^{2}-\beta^{2}\right]}\right. \\
\left.-\frac{1}{\beta^{2}\left[(\omega+i \epsilon)^{2}-\mathbf{k}^{2}\right]}\right\}, \\
\equiv \frac{1}{\beta^{2}}\left[\tilde{g}_{1}(k)-\tilde{g}_{2}(k)\right]
\end{aligned}
$$

which is in fact simply the Fourier transform of Eq. (45). We will analyze each term in Eq. (A-5) separately.

First we need to define the following quantities:

$$
\begin{aligned}
T & \equiv t-t^{\prime}, \\
\mathbf{R} & \equiv \mathbf{r}-\mathbf{r}^{\prime}, \\
\tilde{k} & \equiv|\mathbf{k}|^{2}+\beta^{2} .
\end{aligned}
$$

In what follows, we will set the speed of light $c=1$. The $\tilde{g}_{1}(k)$ term can be integrated as follows:

$$
\begin{aligned}
g_{1 \mathrm{R}}\left(x-x^{\prime}\right) & =\frac{1}{(2 \pi)^{4}} \int \mathrm{d} \omega \mathrm{d}^{3} \mathbf{k} \tilde{g}_{1}(k) \\
& =\frac{1}{4 \pi^{3}} \int \mathrm{d} \omega \mathrm{d}^{3} \mathbf{k} \\
& \times \frac{e^{-i \omega T} e^{i \mathbf{k} \cdot \mathbf{R}}}{[((\omega+i \epsilon)+\sqrt{\tilde{k}})((\omega+i \epsilon)-\sqrt{\tilde{k}})]},
\end{aligned}
$$

where the $+i \epsilon$ above is simply a mnemonic for the retarded Green's function pole prescription. The contour integral in the complex $\omega$ plane results in $-2 \pi i \sum R e s$ and we have

$$
\begin{aligned}
& g_{1 \mathrm{R}}(x-\left.x^{\prime}\right)=\frac{i \Theta(T)}{4 \pi^{2}} \\
& \times \int \mathrm{d}^{3} \mathbf{k} e^{i \mathbf{k} \cdot \mathbf{R}}\left\{\frac{e^{i T \sqrt{\tilde{k}}}-e^{-i T \sqrt{\tilde{k}}}}{\sqrt{\tilde{k}}}\right\} .
\end{aligned}
$$


Upon performing the angular integral, one finds

$$
g_{1 \mathrm{R}}\left(x-x^{\prime}\right)=\frac{\Theta(T) \beta}{2 \pi|\mathbf{R}|}\left[\mathcal{I}^{+}-\mathcal{I}^{-}\right] \text {, }
$$

where

$$
\begin{array}{r}
\mathcal{I}^{ \pm}=\int_{0}^{\infty} \frac{k \mathrm{~d} k}{\sqrt{k^{2}+1}}\left(e^{i \beta\left(|\mathbf{R}| k \pm T \sqrt{k^{2}+1}\right)}\right. \\
\left.+e^{-i \beta\left(|\mathbf{R}| k \pm T \sqrt{k^{2}+1}\right)}\right) .
\end{array}
$$

We will first focus on the solution which is interior to the light cone, i.e., $T>|\mathbf{R}|$. In the first term in the integral, Eq. A-9 above, we perform the following change of variables:

$$
\begin{aligned}
|\mathbf{R}| k+T \sqrt{k^{2}+1} & =\tau \cosh v, \\
T k+|\mathbf{R}| \sqrt{k^{2}+1} & =\tau \sinh v, \\
k & =-\frac{|\mathbf{R}|}{\tau} \cosh v+\frac{T}{\tau} \sinh v, \\
\frac{\mathrm{d} k}{\sqrt{k^{2}+1}} & =\mathrm{d} v,
\end{aligned}
$$

where $v \in\left[v_{0}, \infty\right), v_{0} \equiv \operatorname{arcosh}(T / \tau)$ and we have defined $\tau=\sqrt{T^{2}-|\mathbf{R}|^{2}}$.

In the second term in the integral in Eq. A-9 above, we will perform a different change of variables given by

$$
\begin{aligned}
|\mathbf{R}| k-T \sqrt{k^{2}+1} & =-\tau \cosh \bar{v}, \\
T k-|\mathbf{R}| \sqrt{k^{2}+1} & =\tau \sinh \bar{v}, \\
k & =\frac{|\mathbf{R}|}{\tau} \cosh \bar{v}+\frac{T}{\tau} \sinh \bar{v} . \\
\frac{\mathrm{d} k}{\sqrt{k^{2}+1}} & =\mathrm{d} \bar{v},
\end{aligned}
$$

where for these change of variables $\bar{v} \in\left[-v_{0}, \infty\right)$. Note that, the variable $\bar{v}$ spans the interval $\left[-v_{0}, 0\right]$, while $k$ correspondingly spans the interval $[0,|\mathbf{R}| / \tau]$. After these changes of variables we arrive at the following:

$$
\begin{aligned}
g_{1 \mathrm{R}}\left(x-x^{\prime}\right)= & \frac{\Theta(T) \beta}{2 \pi|\mathbf{R}|}\left\{\int_{v_{0}}^{\infty} \mathrm{d} v\left[-\frac{|\mathbf{R}|}{\tau} \cosh v+\frac{T}{\tau} \sinh v\right]\left[e^{i \beta \tau \cosh v}+e^{-i \beta \tau \cosh v}\right]\right. \\
& \left.-\int_{-v_{0}}^{\infty} \mathrm{d} \bar{v}\left[\frac{|\mathbf{R}|}{\tau} \cosh \bar{v}+\frac{T}{\tau} \sinh \bar{v}\right]\left[e^{i \beta \tau \cosh \bar{v}}+e^{-i \beta \tau \cosh \bar{v}}\right]\right\}, \\
& =\frac{\Theta(T) \beta}{2 \pi|\mathbf{R}|} \int_{-\infty}^{\infty} \mathrm{d} v\left[-\frac{|\mathbf{R}|}{\tau} \cosh v+\frac{T}{\tau} \sinh v\right]\left[e^{i \beta \tau \cosh v}+e^{-i \beta \tau \cosh v}\right] \\
& =-\frac{\Theta(T) \beta}{2 \pi \tau} \int_{-\infty}^{\infty} \mathrm{d} v\left[e^{i \beta \tau \cosh v-v}+e^{-i \beta \tau \cosh v-v}\right],
\end{aligned}
$$

where the last equality follows by symmetry; it should be understood that the limits in the integrals above are such that the integrals are convergent.

We note the following integral representations [22] of the Hankel functions of order $\alpha$ of the first and second kind, respectively:

$$
\begin{aligned}
& H_{\alpha}^{(1)}(x)=\frac{e^{-i \alpha \frac{\pi}{2}}}{\pi i} \int_{-\infty-i \epsilon}^{\infty+i \epsilon} \mathrm{d} v e^{i x \cosh v-\alpha v}, \\
& H_{\alpha}^{(2)}(x)=-\frac{e^{+i \alpha \frac{\pi}{2}}}{\pi i} \int_{-\infty+i \epsilon}^{\infty-i \epsilon} \mathrm{d} v e^{-i x \cosh v-\alpha v},
\end{aligned}
$$

related to the Bessel functions of first and second kind via the relations:

$$
\begin{gathered}
H_{\alpha}^{(1)}(x)=\mathcal{J}_{\alpha}(x)+i \mathcal{Y}_{\alpha}(x), \\
H_{\alpha}^{(2)}(x)=\mathcal{J}_{\alpha}(x)-i \mathcal{Y}_{\alpha}(x),
\end{gathered}
$$

where $\mathcal{J}_{\alpha}(x)$ and $\mathcal{Y}_{\alpha}(x)$ are Bessel functions of the first and second kind respectively; i.e., the two linearly independent solutions to Bessel's equation:

$$
x^{2} \frac{d^{2} F}{d x^{2}}+x \frac{d F}{d x}+\left(x^{2}-\alpha^{2}\right) F=0 .
$$

In particular, we require the Hankel functions of order $\alpha=1$, which are given as

$$
\begin{aligned}
& H_{1}^{(1)}(x)=-\frac{1}{\pi} \int_{-\infty-i \epsilon}^{\infty+i \epsilon} \mathrm{d} v e^{i x \cosh v-v}, \\
& H_{1}^{(2)}(x)=-\frac{1}{\pi} \int_{-\infty+i \epsilon}^{\infty-i \epsilon} \mathrm{d} v e^{-i x \cosh v-v} .
\end{aligned}
$$

Thus, one can express the integral for $g_{1 \mathrm{R}}$, Eq. (A-11, 
in terms of these Hankel functions as

$$
\begin{aligned}
g_{1 \mathrm{R}}\left(x-x^{\prime}\right) & =\frac{\Theta(T) \beta}{2 \pi \tau}\left\{\pi H_{1}^{(1)}(\beta \tau)+\pi H_{1}^{(2)}(\beta \tau)\right\}, \\
& =\Theta(T) \frac{\beta \mathcal{J}_{1}(\beta \tau)}{\tau} \quad \text { for } T>|\mathbf{R}| .(\mathrm{A}-1
\end{aligned}
$$

Note that, $\mathcal{J}_{1}(\beta \tau)$ is the Bessel function of first kind of order 1 and we have used Eqs. (A-12) and (A-13) to arrive at the final expression, Eq. (A-15), above.

Looking now at the exterior of the light cone, i.e., $|\mathbf{R}|>$ $T$, we make the following change in variables in the first term in the integral of Eq. (A-9) above:

$$
\begin{aligned}
|\mathbf{R}||\mathbf{k}|+T \sqrt{|\mathbf{k}|^{2}+1} & =\xi \sinh v, \\
T|\mathbf{k}|+|\mathbf{R}| \sqrt{|\mathbf{k}|^{2}+1} & =\xi \cosh v, \\
k & =\frac{|\mathbf{R}|}{\xi} \sinh v-\frac{T}{\xi} \cosh v, \\
\frac{\mathrm{d}|\mathbf{k}|}{\sqrt{|\mathbf{k}|^{2}+1}} & =\mathrm{d} v .
\end{aligned}
$$

where as before $v \in\left[v_{0}, \infty\right)$, but now $v_{0} \equiv \operatorname{arsinh}(T / \xi)$ and we have defined $\xi=\sqrt{|\mathbf{R}|^{2}-T}$.

In the second term in the integral of Eq. (A-9) we perform the following change of variables:

$$
\begin{aligned}
|\mathbf{R}||\mathbf{k}|-T \sqrt{|\mathbf{k}|^{2}+1} & =\xi \sinh \bar{v}, \\
-T|\mathbf{k}|+|\mathbf{R}| \sqrt{|\mathbf{k}|^{2}+1} & =\xi \cosh \bar{v}, \\
k & =\frac{|\mathbf{R}|}{\xi} \sinh \bar{v}+\frac{T}{\xi} \cosh \bar{v}, \\
\frac{\mathrm{d}|\mathbf{k}|}{\sqrt{|\mathbf{k}|^{2}+1}} & =\mathrm{d} \bar{v},
\end{aligned}
$$

where $\bar{v} \in\left[-v_{0}, \infty\right)$.

Upon using these change of variables we arrive at

$$
g_{1 \mathrm{R}}\left(x-x^{\prime}\right)=-2 \frac{\Theta(T)}{|\mathbf{R}|} \frac{T}{\xi} \delta\left(\sqrt{|\mathbf{R}|^{2}-T^{2}}\right),
$$

which vanishes since we are explicitly considering the $|\mathbf{R}|>T$ region and thus the delta function vanishes.

We still have yet to determine the singular part of the Green's function on the light cone $|\mathbf{R}|=T$. To do this we will repeat the formalism established in Ref. [21]. Note that, we very well could have determined the full Green's function $g_{1 \mathrm{R}}\left(x-x^{\prime}\right)$ and not just the part on the light cone via the formalism in Ref. [21]. In fact, a full review of the formalism will serve as a useful check on the calculation of the smooth part of the Green's function determined above via Fourier transform.

To begin, we first integrate the Green's function equation, Eq. (A-1), over a space-time volume which contains the source event $x^{\prime}=0$, namely

$$
\int_{\partial V}\left(g_{1}\right)^{; \mu} \mathrm{d} \Sigma_{\mu}-\beta^{2} \int_{V} g_{1}=4 \pi,
$$

where Gauss' theorem was used to arrive at the first term above and $d \Sigma_{\mu}$ is the surface element of the boundary $\partial V$. Assuming $\int_{V} f_{1}$ vanishes as the integration volume vanishes, we are left with

$$
\lim _{V \rightarrow 0} \int_{\partial V}\left(g_{1}\right)^{; \mu} \mathrm{d} \Sigma_{\mu}=4 \pi .
$$

Now introduce the coordinates $(w, \chi, \theta, \phi)$ given by

$$
\begin{aligned}
t & =w \cos \chi, \\
x & =w \sin \chi \sin \theta \cos \phi, \\
y & =w \sin \chi \sin \theta \sin \phi, \\
z & =w \sin \chi \cos \theta,
\end{aligned}
$$

such that the line element $\mathrm{d} s^{2}=g_{\alpha \beta} \mathrm{d} x^{\alpha} \mathrm{d} x^{\beta}$ of the flat background takes the form

$$
\begin{aligned}
\mathrm{d} s^{2}= & -\cos 2 \chi \mathrm{d} w^{2}+2 w \sin 2 \chi \mathrm{d} w \mathrm{~d} \chi \\
& +w^{2} \cos 2 \chi \mathrm{d} \chi^{2}+w^{2} \sin ^{2} \chi \mathrm{d} \Omega^{2},
\end{aligned}
$$

where $\mathrm{d} \Omega^{2}=\mathrm{d} \theta^{2}+\sin ^{2} \theta \mathrm{d} \phi^{2}$. In these coordinates, the surface $\partial V$ is given by constant $w$, and the Synge world function $\sigma$ is

$$
\sigma=-\frac{1}{2} w^{2} \cos 2 \chi
$$

notice that for time-like events $-2 \sigma=\tau^{2}$ where $\tau$ is as previously defined.

The following quantities will be useful for what follows:

$$
\begin{aligned}
\sqrt{-g} & =w^{3} \sin ^{2} \chi \sin \theta, \\
g^{w w} & =-\cos 2 \chi, \\
g^{w \chi} & =\frac{\sin 2 \chi}{w} \\
g^{\chi \chi} & =\frac{\cos 2 \chi}{w^{2}}
\end{aligned}
$$

where $g$ is the determinant of the metric $g_{\alpha \beta}$. The only nonzero component of $\mathrm{d} \Sigma_{\alpha}$ is

$$
\mathrm{d} \Sigma_{w}=w^{3} \sin ^{2} \chi \mathrm{d} \chi \mathrm{d} \Omega,
$$

where $\mathrm{d} \Omega=\sin \theta \mathrm{d} \theta \mathrm{d} \phi$.

In these coordinates, the retarded Green's function is given by:

$$
g_{1 \mathrm{R}}=\Theta(w \cos \chi) g(\sigma),
$$

where $g(\sigma)$ is an as yet undetermined, possibly distributional, function. We will only need the following gradient of $g_{1 \mathrm{R}}$ (omitting the label $\mathrm{R}$ )

$$
\begin{aligned}
\left(g_{1}\right)^{; w} & =g^{w \mu}\left(g_{1}\right)_{; \mu} \\
& =g^{w w}\left(g_{1}\right)_{; w}+g^{w \chi}\left(g_{1}\right)_{; \chi} .
\end{aligned}
$$


A straight-forward calculation leads to

$$
\begin{aligned}
\left(g_{1}\right)^{; w}= & -\delta(w \cos \chi) \cos \chi g(\sigma) \\
& +w \Theta(w \cos \chi) g^{\prime}(\sigma),
\end{aligned}
$$

where the prime on $g(\sigma)$ denotes differentiation with respect to $\sigma$. We then have

$$
\begin{aligned}
\int_{\partial V}\left(g_{1}\right)^{; \mu} \mathrm{d} \Sigma_{\mu}= & \int_{\partial V} w^{3} \sin ^{2} \chi \mathrm{d} \chi \mathrm{d} \Omega\left\{w \Theta(w \cos \chi) g^{\prime}(\sigma)\right. \\
& -\delta(w \cos \chi) \cos \chi g(\sigma)\} \\
= & 4 \pi w^{4} \int_{0}^{\frac{\pi}{2}} \sin ^{2} \chi \mathrm{d} \chi g^{\prime}(\sigma), \quad(\mathrm{A}-25)
\end{aligned}
$$

where the Heavyside function has restricted the limits of $\chi$ integration such that $\cos \chi \geq 0$ and the delta term vanishes.

Changing integration variable from $\chi$ to $\sigma$ in the integral above we arrive at the following condition on $g(\sigma)$ :

$$
\lim _{\epsilon \rightarrow 0} \epsilon \int_{-\epsilon}^{\epsilon} \mathrm{d} \sigma \Xi\left(\frac{\sigma}{\epsilon}\right) g^{\prime}(\sigma)=1
$$

where

$$
\begin{gathered}
\epsilon \equiv \frac{1}{2} w^{2}, \\
\Xi\left(\frac{\sigma}{\epsilon}\right) \equiv \sqrt{\frac{1+\frac{\sigma}{\epsilon}}{1-\frac{\sigma}{\epsilon}} .}
\end{gathered}
$$

We now propose the following ansatz for $g(\sigma)$ :

$$
\begin{aligned}
g(\sigma)= & V(\sigma) \Theta(-\sigma)+A \delta(\sigma)+B \delta^{\prime}(\sigma) \\
& +C \delta^{\prime \prime}(\sigma)+D \delta^{\prime \prime \prime}(\sigma)+\ldots
\end{aligned}
$$

where $V(\sigma)$ is a smooth function of $\sigma$ and $A, B, \ldots$ are constants. Inserting the ansatz Eq. A-27 into Eq. (A-26) gives

$$
\lim _{\epsilon \rightarrow 0} \epsilon\left\{\int_{-\epsilon}^{\epsilon} \mathrm{d} \sigma \Xi\left(\frac{\sigma}{\epsilon}\right) V^{\prime}(\sigma) \Theta(-\sigma)-\Xi(0) V(0)-\frac{A}{\epsilon} \dot{\Xi}(0)+\frac{B}{\epsilon^{2}} \ddot{\Xi}(0)-\frac{C}{\epsilon^{3}} \dddot{\Xi}(0)+\frac{D}{\epsilon^{4}} \Xi^{(4)}(0)+\ldots\right\}=1 .
$$

The first two terms on the left-hand side of Eq. (A-28) above vanish, since $V(\sigma)$ is assumed to be smooth. The limit exist as long as $B=C=\ldots=0$ and the condition Eq. (A-26) will be satisfied iff $A=-1$, since $\dot{\Xi}(0)=1$. To fully determine the smooth part of $g(\sigma)$ one needs then only to solve the homogeneous equation (i.e., $x \neq$ $\left.x^{\prime}\right)$ :

$$
\left(\square-\beta^{2}\right) g(\sigma)=4 g^{\prime}(\sigma)+2 \sigma g^{\prime \prime}(\sigma)-\beta^{2} g(\sigma)=0,
$$

from which it is straight forward to verify that (the reader is referred to Ref. [21] for further details)

$$
V(\sigma)=\frac{\beta \mathcal{J}_{1}(\beta \sqrt{-2 \sigma})}{\sqrt{-2 \sigma}}
$$

Returning to the original coordinates $(t, r, \theta, \phi)$ we then have

$$
\begin{aligned}
g_{1 \mathrm{R}}\left(x-x^{\prime}\right)= & -\delta\left(\frac{1}{2} \tau^{2}\right) \Theta(T) \\
& +\frac{\beta \mathcal{J}_{1}(\beta \tau)}{\tau} \Theta\left(\frac{1}{2}\left(T^{2}-|\mathbf{R}|^{2}\right)\right) \Theta(T), \\
= & -2 \delta\left(\tau^{2}\right) \Theta(T) \\
& +\frac{\beta \mathcal{J}_{1}(\beta \tau)}{\tau} \Theta(T-|\mathbf{R}|) \Theta(T), \quad(\mathrm{A}-30)
\end{aligned}
$$

where we have used some standard properties of the Dirac delta function and the Heavyside step function.

To determine $g_{2 \mathrm{R}}\left(x-x^{\prime}\right)$ we need to consider the integral

$$
\begin{aligned}
g_{2 \mathrm{R}}\left(x-x^{\prime}\right)=\frac{1}{4 \pi^{3}} & \int \mathrm{d} \omega \mathrm{d}^{3} \mathbf{k} \\
& \times \frac{e^{-i \omega T} e^{i \mathbf{k} \cdot \mathbf{R}}}{[(\omega+i \epsilon+|\mathbf{k}|)(\omega+i \epsilon-|\mathbf{k}|)]},
\end{aligned}
$$

where the pole prescription is again that of the retarded Green's function. A straight-forward calculation leads to

$$
\begin{aligned}
g_{2 \mathrm{R}}\left(x-x^{\prime}\right) & =\frac{\Theta(T)}{|\mathbf{R}|}\{\delta((T+|\mathbf{R}|)-\delta(T-|\mathbf{R}|)\} \\
& =-2 \Theta(T) \delta\left(\tau^{2}\right) .
\end{aligned}
$$

Finally, the Green's function which satisfies the fourthorder wave equation Eq. (42) is given by

$$
\begin{aligned}
G_{\mathrm{R}}\left(x-x^{\prime}\right) & =\frac{1}{\beta^{2}}\left(g_{1 \mathrm{R}}\left(x-x^{\prime}\right)-g_{2 \mathrm{R}}\left(x-x^{\prime}\right)\right), \\
& =\frac{\beta \mathcal{J}_{1}(\beta \tau)}{\beta \tau} \Theta(T-|\mathbf{R}|) \Theta(T)
\end{aligned}
$$

Note that the Green's function above is subject to the 
initial value condition:

$\left.\frac{\partial G_{\mathrm{R}}\left(x-x^{\prime}\right)}{\partial t}\right|_{t=0}=0$. 